\title{
Changes in proviral load (PVL) among patients with rapidly progressive HTLV-1-associated myelopathy/ tropical spastic paraparesis (HAM/TSP) receiving empirical therapy
}

\author{
Eduardo Gotuzzo ${ }^{1,2,3^{*}}$, Carolina Alvarez ${ }^{1}$, Elsa González ${ }^{1,2}$, Martin A Tipismana ${ }^{1}$, Giovanni M López ${ }^{1}$, \\ Kristien Verdonck ${ }^{1,4}$, Daniel Clark ${ }^{1,5}$
}

From 15th International Conference on Human Retroviruses: HTLV and Related Viruses

Leuven and Gembloux, Belgium. 5-8 June 2011

\section{Introduction}

Previous studies suggest that HTLV-1 PVL remains constant over time. We compare PVL, at baseline and follow-up, in patients with rapidly progressive HAM/TSP who received empirical treatment.

\section{Methods}

Rapidly progressive HAM/TSP was defined by patients' incapacity to walk unaided within two years after symptoms' onset. These cases are treated at our center with prednisone in combination with Lamivudine and/or Zidovudine at standard doses. PVL were performed by duplicate in PBMC of blood samples drawn before (baseline) and during treatment; values are reported in copies $/ 10^{4}$ PBMC.

\section{Results}

We evaluated 11 patients (6 women); their median age was 50 years-old, interquartile range [IQR] 20, with significant differences between men and women (medians: 56 and 36 , respectively, $\mathrm{p}=0.02$ ). Median HAM/TSP duration at diagnosis was 12 months (IQR 4). The median time (all in months) between the symptoms onset and the beginning of therapy was 14 (IQR: 9); between HAM/TSP onset and the baseline PVL, 14 (IQR: 13); between PVL determinations, 7 (IQR: 7). Median PVL values were 1183 (IQR: 1425) at baseline, and 1002 at follow-up (IQR: 1425). In the group of $7 / 11$ patients where PVL decreased, differences between baseline and follow-up determinations were significant $(\mathrm{p}=0.01)$, with a median paired reduction of 362 (IQR: 1425). In the four cases where PVL increased, differences were not significant $(\mathrm{p}=0.06)$.

\section{Conclusions}

In most of the cases here reported, PVL decreased significantly during empirical therapy. Clinical correlates and long-term evaluation of these findings demand controlled therapy trials.

\section{Author details \\ ${ }^{1}$ Instituto de Medicina Tropical Alexander von Humboldt, Universidad Peruana Cayetano Heredia, Lima, Perú. ${ }^{2}$ Facultad de Medicina, Universidad Peruana Cayetano Heredia, Lima, Perú. ${ }^{3}$ Departamento de Enfermedades Infecciosas, Tropicales y Dermatología, Hospital Nacional Cayetano Heredia, Lima, Perú. ${ }^{4}$ Institute of Tropical Medicine, Antwerp, Belgium. ${ }^{5}$ Laboratorios de Investigación y Desarrollo (LID), Facultad de Ciencias y Filosofía, Universidad Peruana Cayetano Heredia, Lima, Perú.}

Published: 6 June 2011

doi:10.1186/1742-4690-8-S1-A58

Cite this article as: Gotuzzo et al:: Changes in proviral load (PVL) among patients with rapidly progressive HTLV-1-associated myelopathy/tropical spastic paraparesis (HAM/TSP) receiving empirical therapy. Retrovirology 2011 8(Suppl 1):A58.

\footnotetext{
* Correspondence: eduardo.gotuzzo@upch.pe

IInstituto de Medicina Tropical Alexander von Humboldt, Universidad

Peruana Cayetano Heredia, Lima, Perú

Full list of author information is available at the end of the article
}

(c) 2011 Gotuzzo et al; licensee BioMed Central Ltd. This is an open access article distributed under the terms of the Creative Commons 